\title{
COMPARISON OF REGULAR AND INVERTED BATCH DISTILLATION
}

\author{
EVA SøRENSEN* and SIGURD SKOGESTAD \\ Department of Chemical Engineering, Norwegian University of Science and Technology, NTNU, \\ N-7034 Trondheim, Norway
}

(Received 2 September 1994; accepted 4 April 1996)

\begin{abstract}
Several alternative strategies have been suggested to increase the productivity of batch distillation. One is the use of inverted columns where the feed is charged to the condenser drum instead of to the reboiler as for regular columns. In the first part of this paper, optimal results in terms of minimum operating time for separations in a regular and an inverted batch column are presented. The differences in dynamic behaviour between the two columns are discussed. Intuitively, one may think that an inverted column is best for separations with large amounts of light component in the feed since, in this case, only a small amount of heavy component needs to be removed from the column. Surprisingly, the opposite is found: the inverted column is better in terms of less operating time when the light component is present in only small amounts and where a large amount of heavy component is removed from the column. In the second part of the paper inverted separations are discussed; i.e. removal of light component in a regular column vs removal of heavy component in an inverted column. Finally, a qualitative comparison between a regular column and an ideal inverted column, where the feed is in vapour phase, is presented. Copyright (C) 1996 Elsevier Science Ltd
\end{abstract}

Keywords: Batch, distillation, configurations, optimization, regular, inverted.

\section{INTRODUCTION}

Batch distillation is a very old unit operation in the chemical industry. During the last century, continuous distillation took over most of the processes where batch distillation was originally used. However, batch processes are becoming increasingly important again as a result of the expansion in the fine chemical and pharmaceutical industries. These industries are characterised by small amounts of products with high added value. Batch distillation has the advantage of being able to separate a number of products in a single column. A batch column can handle (1) multicomponent mixtures, (2) a wide range of feed compositions and (3) a varying degree of difficulty of separation (wide range of relative volatilities and product purities). The demand for efficiency and productivity in the chemical industry is continuously increasing. However, the optimal operation of a unit is limited by the given operating strategy and the process equipment. Therefore there is a need for new or alternative strategies which can increase the productivity.

The use of inverted batch columns or 'regular batch columns turned upside down' has been suggested earlier in the literature. In the inverted case, the feed is charged to the condenser drum and further continuously added to the top of the column. The liquid from

*Corresponding author at: Department of Chemical and Biochemical Engineering, University College London, Torrington Place, London WC1E 7JE, U.K. Tel.: + 44-171-4193802. Fax: + 44-171-383-2348. E-mail: e.sorensen@ucl. ac.uk. the bottom of the column is vaporised in the reboiler and a fraction is removed as product. The products are taken out with the heaviest component first, then the second heaviest, etc. The true inverse of a regular batch column requires both the feed charge stored in the condenser drum and the product taken out to be in vapour phase which is very unlikely. It is therefore normally assumed that the feed and the products are in liquid phase. However, this configuration is not the true inverse of the regular column as we will show in this paper.

The use of an inverted, or stripping, column was originally proposed by Robinson and Gilliland (1950). They stated that the main advantage with this configuration was that the most volatile components would be collected in the condenser drum in high purity. They briefly discussed the possibility of running the column first in a normal batch fashion to remove the lighter components and then inverted to remove the heavier constituents. They also discussed a combined operation where the feed was added to the middle of the column and the light and heavy components were taken off simultaneously over the top and bottom, respectively.

The combined operation was studied by Hasebe et al. (1992) who denoted this column configuration a complex column. They also discussed the difference between regular and inverted columns. They claimed that a regular column always has a better separation efficiency than an inverted column if the separation conditions are the same and the relative volatility is constant (what we call 'inverted separations' in this paper). The conclusion was based on comparison between the equilibrium curves and operation lines for 
a regular and an inverted column with all other conditions equal. However, we will in this paper show that this is not always the case.

Mujtaba and Macchietto (1992) discussed the use of a complex column to improve the operation of reactive batch distillation. They found that this column configuration improved conversion and product yield significantly when the reaction products had two extreme boiling points (highest and lowest in the reaction mixture). The use of an inverted column for cases where the reaction product had a higher boiling point than the reactants was suggested but no examples were given. Mujtaba and Macchietto (1994) discussed the use of inverted and complex columns for an example with reactive batch distillation. For this example the inverted column gave a lower conversion than the regular column. This was explained in terms of the difference in relative volatility between the heavy components compared to between the light ones.

Chiotti and Iribarren (1991) presented simplified models for binary batch distillation in both regular and inverted columns. They included an intermediate cut which was recycled to the next feed charge. Their models were based on the assumption of pseudosteady-state in the column. Numerical integration was thereby avoided. They stated that the inverted column was more economical than the regular one for heavy products of high purity. They presented two numerical examples where they optimised the total annual cost for two separations in both an inverted and a regular column. Chiotti et al. (1993) extended these models to multicomponent mixtures. They also considered columns which could be used for both rectification (regular column) and stripping (inverted column) where the products were taken off both at the top and the bottom of the column in given sequences. With their very simplified models they found that, for a ternary mixture, the inverted column was optimal when the purity specification for the heavy product was high. It should be noted that both in this paper and in the previous one, the optimal number of trays in the column was in most cases higher for the inverted column than for the regular one.

To the best of our knowledge, no real comparison between regular and inverted columns in terms of optimal operation has been given in the literature so far.

This paper is naturally divided into two parts. In the first part, we consider the optimal operation of a regular and an inverted batch column in terms of minimum operating time for a number of separations (Section 3). Thereafter we discuss the differences in dynamic behaviour between the two columns (Section 4). Intuitively, one may think that an inverted column is best for separations with a large amount of light component in the feed, because one may somehow avoid to 'boil off' this large amount. However, as we shall see, the opposite conclusion is true: the inverted column is preferable to the regular column, in terms of shorter operating time, when the light com- ponent is present in small amounts in the feed mixture. In the second part of the paper (Section 5), we discuss the operation of the two columns for inverted separations. By inverted separations is meant the removal of light component in a regular column vs removal of heavy component in an inverted column. Finally, a qualitative comparison between a regular batch column and an ideal inverted column where the feed is in vapour phase is presented.

\section{DYNAMIC MODEL}

In this paper we compare the operation of a regular and an inverted batch distillation column. A schematic representation of the two columns is given in Fig. 1. Note that both the feed and the product are in liquid phase also in the inverted column. The ideal inverted column with feed and product in vapour phase is discussed at the end of this paper.

We limit the discussion to a binary mixture with constant relative volatility $\alpha=2$. The columns are assumed to have $N=10$ theoretical trays, the initial feed is $H_{F}=10 \mathrm{kmol}$ and the vapour flow from the reboiler is $V_{B}=10 \mathrm{kmol} / \mathrm{h}$. It is. assumed that the vapour flow $V_{B}$ can be manipulated directly. The tray holdups are $H_{j}=0.001 \mathrm{kmol}$, the condenser holdup for the regular column is $H_{c}=0.01 \mathrm{kmol}$ and the reboiler holdup for the inverted column is $H_{B}=0.01 \mathrm{kmol}$. In the following we will compare cases with different feed compositions and product purity specifications. It is assumed that in the regular column, the feed is charged to the reboiler and in the inverted column to the condenser drum. In both cases the feed is liquid at its boiling point. The time and energy input to heat the feed charge to its boiling point is neglected. In any case this time is the same for the two columns since the feed is in liquid phase in both cases, but for an ideal inverted column the feed charge has to be completely evaporated. Initially, the column is assumed filled with liquid at its boiling point with a composition equal to that of the feed. [This assumption is not important for the results, see Sørensen (1994).] The dynamic models for the regular and the inverted column are given in the Appendix. SPEEDUP (1993) is used for the simulations.

\section{OPTIMAL OPERATION}

In this section we consider the optimal operation of both a regular and an inverted column in terms of minimum operating time for given separations. We will show that the inverted column is better than the regular column for separations where the light component is present in a small amount in the feed mixture.

\subsection{The minimum time problem}

The optimal operation in terms of minimum operating time can be stated as:

$$
\min _{R, R_{B}} t_{f}
$$




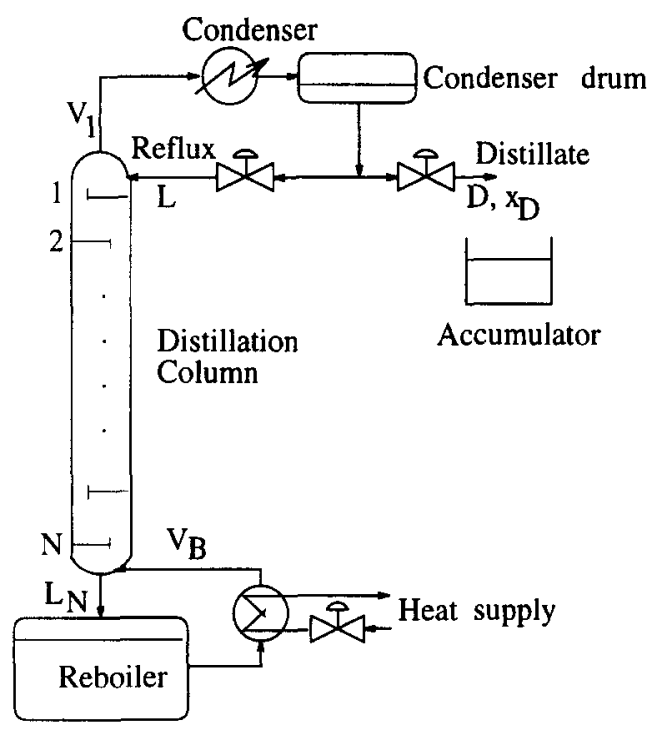

REGULAR COLUMN

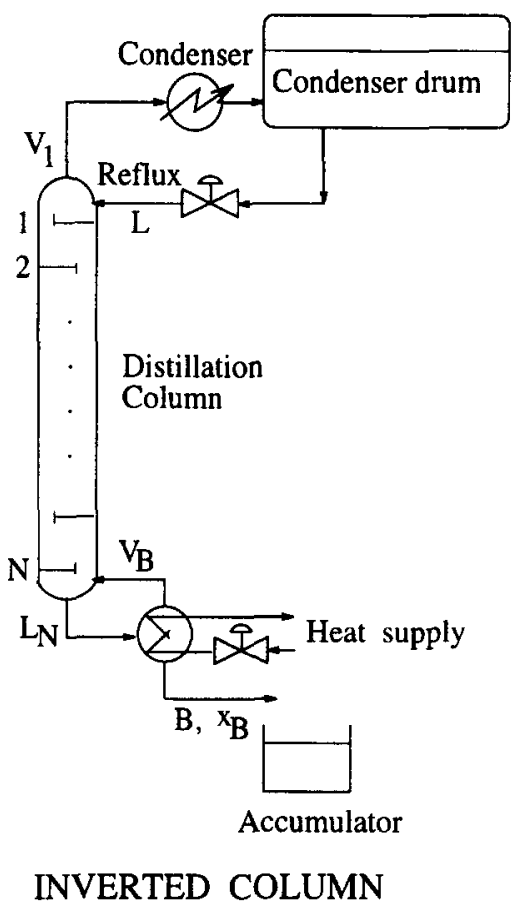

Fig. 1. A regular and an inverted batch distillation column.

subject to

$$
\begin{aligned}
& x_{A}\left(t_{f}\right) \geqslant x_{A}^{\mathrm{spec}} \\
& x_{R}\left(t_{f}\right) \geqslant x_{R}^{\mathrm{spec}} .
\end{aligned}
$$

In words, find the optimal reflux ratio $R$ (for the regular column) or reboiler ratio $R_{B}$ (for the inverted column) which minimises the total operating time $t_{f}$ subject to constraints on the compositions of accumulated light and heavy product at the final time, $x_{A}\left(t_{f}\right)$ and $x_{R}\left(t_{f}\right)$. The light product is in the accumulator and the heavy product in the column and reboiler for the regular column. For the inverted column, the light product is in the condenser and column and the heavy product is in the accumulator. In the following examples, we have chosen to use the same purity specifications for the two products, that is

$$
x_{A}^{\text {spec }}=1-x_{R}^{\text {spec }}=x^{\text {spec }} \text {. }
$$

The product specifications are met simultaneously since no offcuts are produced.

The optimisation variables $R$ and $R_{B}$ are assumed piecewise continuously linear over two intervals ( $t_{0}$ to $t_{1}$ and $t_{1}$ to $\left.t_{f}\right)$ giving a total of five optimisation variables $\left[R\left(t_{0}\right), R\left(t_{1}\right), R\left(t_{f}\right), t_{1}\right.$ and $\left.t_{f}\right]$. It should be noted that several local minima may exist but only the best optimal solution is presented in the following. The optimisation program DAEOPT developed at Imperial College, London (Vassiliadis, 1993), is used where the solution of the optimal control problem is based on control vector parameterisation. The solution method includes two levels. The first level performs an integration of the differential and algebraic equations for fixed values of the optimisation variables. The second level optimises these variables and satisfies end point constraints. A complete solution of the dynamic model for each trial value of the optimisation variables is required.

\subsection{Results}

We consider 13 separations with different feed compositions and product specifications. Other operating parameters are as given in Section 2. The minimum operating time is presented for the 13 cases in Table 1 and shown graphically in Fig. 2. The upper bound on the operating time is $100 \mathrm{~h}$.

From Table 1 and Fig. 2 it can be seen that the inverted column has indeed a shorter operating time than the regular column for some separations. More surprisingly, the inverted column is better for cases where the products are to be recovered at high purity from a feed low in light component, whereas the regular column is found to be best for cases where the products are to be recovered at high purity from a feed rich in light component. The regular column is found to be better than the inverted column for all the symmetrical separations, $x_{F}=0.5$. However, more importantly, for some specifications the inverted column gives a moderate operating time when the separation is not even possible in the regular column $\left(x_{F}=0.1\right.$ and $x^{\text {spec }}=0.999$ ).

The optimal reflux (regular column) and reboiler (inverted column) ratios are shown for three of the cases in Fig. 3. Note that the ratios are defined as internal ratios; $R=L / V_{1}$ for the regular column and $R_{B}=V_{B} / L_{N}$ for the inverted column. The reflux and reboiler ratios are very close to 1 for separations where a small amount of product is to be withdrawn 
from the column leaving a large amount of the other product as residue (e.g. $x_{F}=0.1$ in the regular column and $x_{F}=0.9$ in the inverted column). In the opposite case, where a large amount is withdrawn, the ratios

Table 1. Minimum operating time $t_{f}$ for varying product purity specifications $x^{\text {spec }}$ for regular and inverted column (same purity specification $x^{\mathrm{spec}}$ for both products)

\begin{tabular}{ccc}
\hline & \multicolumn{2}{c}{ Minimum operating time } \\
$\begin{array}{c}\text { Purity } \\
\text { specification } \\
x^{\text {spec }}\end{array}$ & $\begin{array}{c}\text { inverted column } \\
t_{f}, h r\end{array}$ & $\begin{array}{c}\text { regular column } \\
t_{f}, h r\end{array}$ \\
& Feed composition $x_{F}=0.1$ \\
0.925 & 1.043 \\
0.950 & 1.369 & $\mathbf{0 . 4 0 9}$ \\
0.975 & $\mathbf{2 . 2 6 4}$ & $\mathbf{1 . 0 2 4}$ \\
0.980 & $\mathbf{2 . 5 3 2}$ & 2.940 \\
0.990 & $\mathbf{4 . 0 4 1}$ & 4.448 \\
& & $>100$ \\
0.925 & Feed composition $x_{F}=0.5$ & \\
0.950 & 3.022 & $\mathbf{2 . 2 0 0}$ \\
0.990 & 3.807 & $\mathbf{2 . 6 1 0}$ \\
& 10.010 & $\mathbf{5 . 9 0 5}$ \\
0.925 & Feed composition $x_{F}=0.9$ & \\
0.950 & $\mathbf{0 . 7 0 9}$ & 1.248 \\
0.975 & 1.791 & $\mathbf{1 . 6 3 0}$ \\
0.980 & 4.691 & $\mathbf{2 . 4 1 0}$ \\
0.990 & 7.856 & $\mathbf{2 . 4 6 2}$ \\
& $>100$ & $\mathbf{3 . 1 9 2}$ \\
\hline
\end{tabular}

are low initially but increase with time as the column is gradually depleted of the withdrawn product (e.g. $x_{F}=0.9$ in the regular column and $x_{F}=0.1$ in the inverted column).

The compositions of the products in the residual and the accumulator are given in Fig. 4 for one case when the inverted column is best $\left(x_{F}=0.1\right.$ and $x^{\mathrm{spec}}=0.98$ ). For the regular column, the accumulator composition is slightly higher than the specification during most of the run and reaches the specified value at the final time. In the inverted column, the composition in the residue is slowly increasing as the heavy component is removed in the bottom and reaches the specification at the final time.

The relative time saving of the inverted column over the regular column is given in Fig. 5 for varying holdups and relative volatilities for the case with $x_{F}=0.1$. The time saving is given as

$$
\text { time saving }=\frac{t_{f}(\text { regular })-t_{f}(\text { inverted })}{t_{f}(\text { inverted })} .
$$

From the top plot it can be seen that the inverted column becomes more favourable for increasing holdups: as the holdup increases the relative time saving becomes larger for a given purity specification. Also the inverted column becomes more favourable over a wider purity region. The same tendency is found for decreasing relative volatilities (Fig. 5, bottom plot).
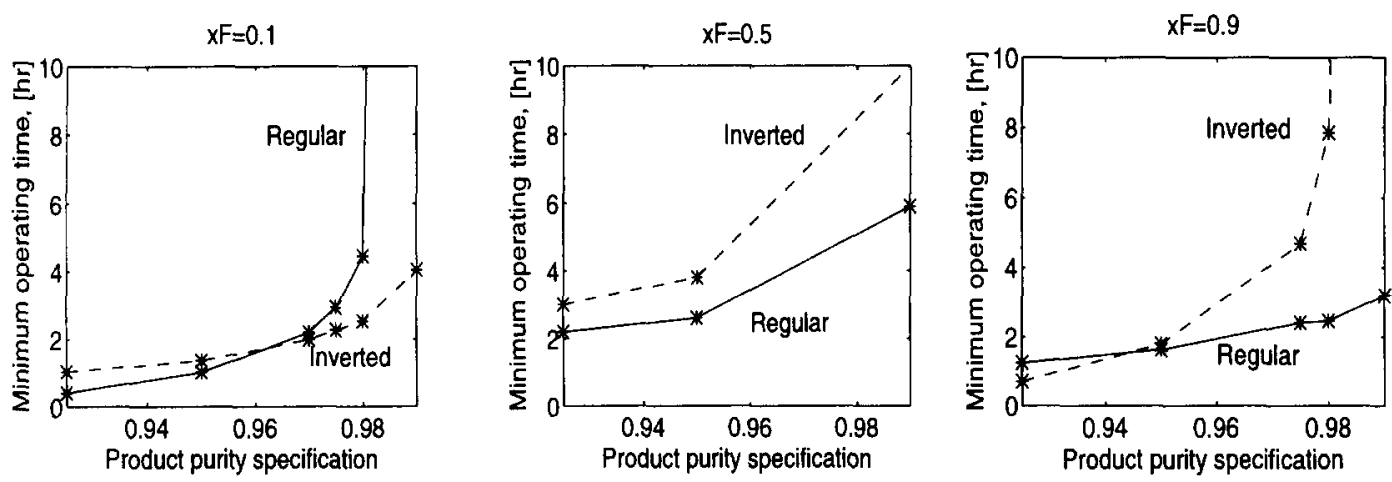

Fig. 2. Minimum operating time $t_{f}$ as a function of product purity specifications $x^{\text {spec }}$ for a regular and an inverted column (same purity specification $x_{\text {spec }}$ for both products).
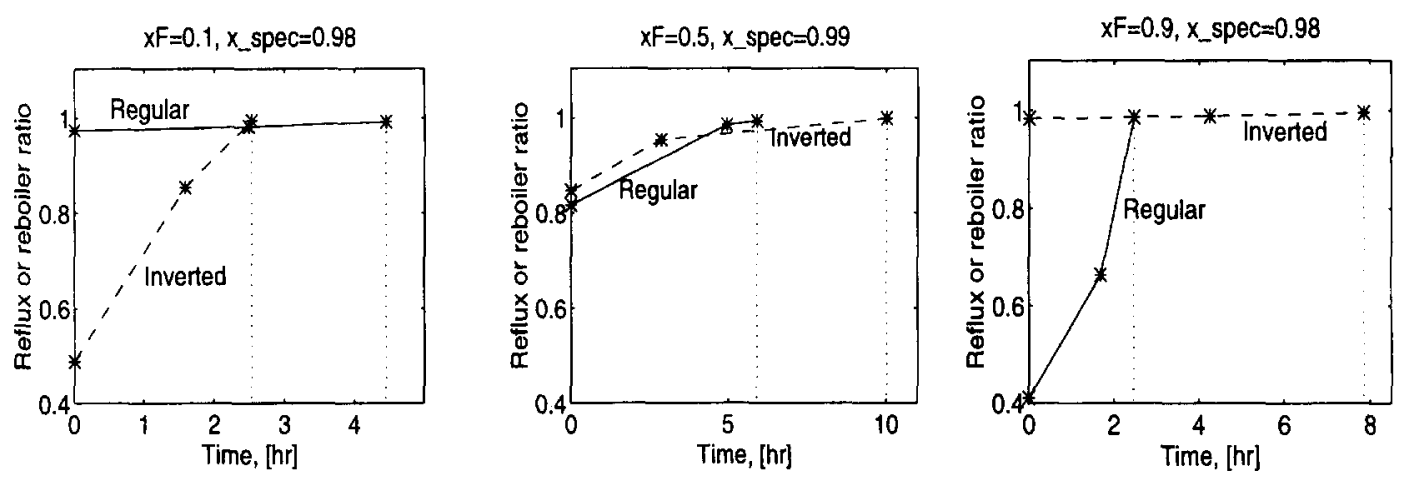

Fig. 3. Optimal reflux ratio $R$ (regular column) and reboiler ratio $R_{B}$ (inverted column). 

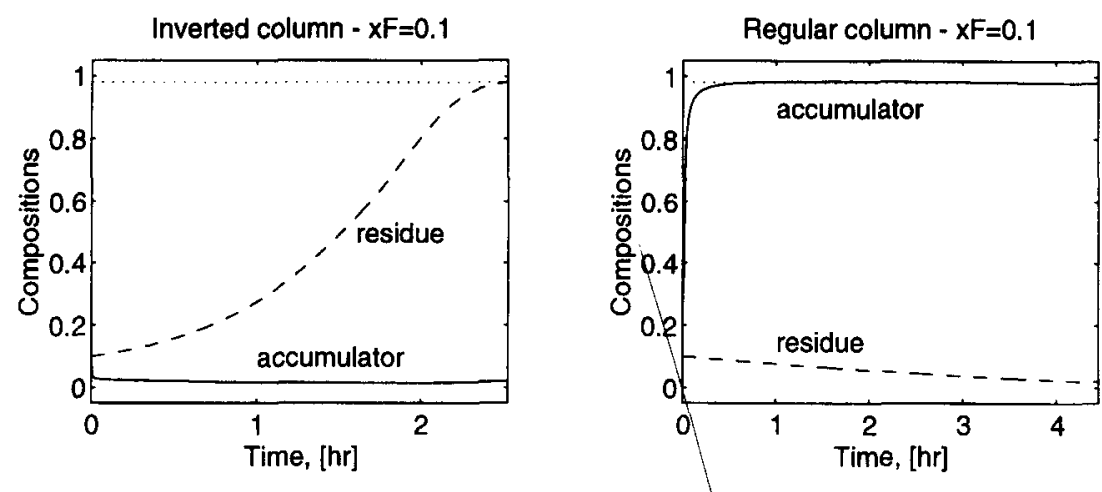

Fig. 4. Composition of product in accumulator and residue $\left(x_{F}=0.1\right.$ and $\left.x^{\text {spec }}=0.98\right)$. Inverted column: light component in residue, heavy component in accumulator. Regular column: light component in accumulator, heavy component in residue.

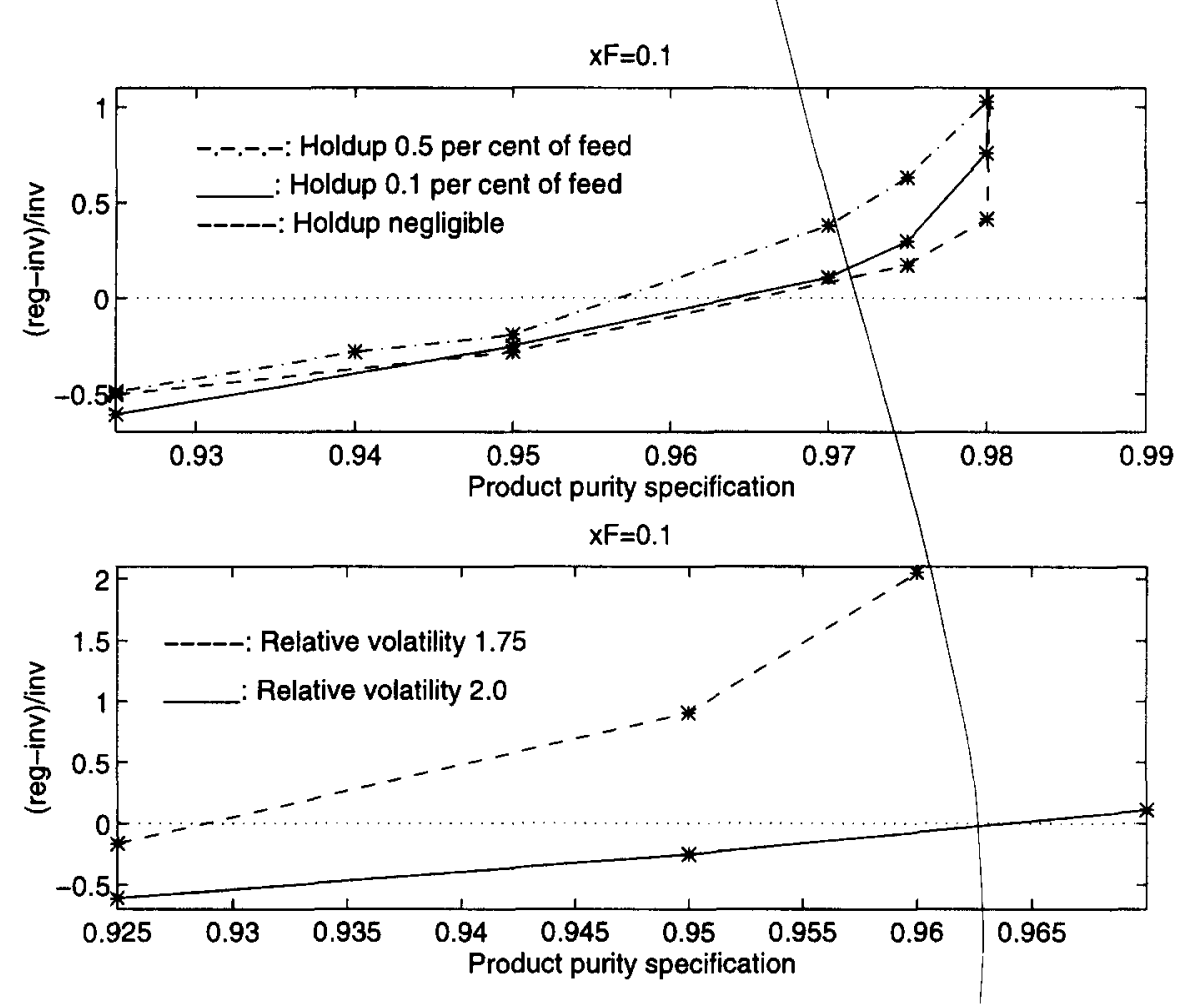

Fig. 5. Relative time saving for varying holdups (top) and relative volatilities (bottom) as a function of purity specification $\left(x_{F}=0.1\right)$.

\subsection{Discussion}

Intuitively, one may think that an inverted column is best for separations with a large amount of light component in the feed, because one may somehow avoid to 'boil off' this large amount. The small amount of heavy component is taken out in the bottom and the large amount of light component remains in the column. However, in the previous section we have found the opposite to be true: the inverted column is preferable to the regular column, in terms of shorter operating time, when the light component is present in small amounts in the feed mixture. The main reason for this is that when a small amount of component is to be removed from the column at high purity, a very high reflux or reboiler ratio must be used in order to meet the product specifications. Consider for instance the case with $x_{F}=0.1$ and $x_{\text {spec }}=0.98$. In order to remove the light component from the feed in a regular column a very high reflux ratio is needed (Fig. 3, left plot). For the inverted column, however, a large amount of heavy component is removed from the column and the light component is accumulated in the column and condenser. The reboiler ratio can initially be very low but is increased gradually as the column is depleted of the heavy component (Fig. 3, left plot). The same is true in the opposite case when the feed is rich in light component. The reboiler ratio for the inverted column is very high while the reflux ratio for the inverted column is low to moderate 
(see the case with $x_{F}=0.9$ and $x_{\text {spec }}=0.98$ in Fig. 3, right plot).

In conclusion, when high purity is required, it is more time consuming to remove a small amount of one component from a column using a high reflux or reboiler ratio than to remove a large amount of the other component using a low to moderate ratio with the opposite column configuration. When only low purity is specified, the opposite is true (e.g. the case with $x_{F}=0.1$ and $x_{\text {spec }}=0.925$ when the regular column is best or the case with $x_{F}=0.9$ and $x_{\text {spec }}=0.925$ when the inverted column is best).

Note that the results are independent of holdup. The same conclusions are reached when the holdup in the column section and either the condenser holdup (regular column) or the reboiler holdup (inverted column) are assumed negligible (see Fig. 5).

\subsection{Practical considerations}

For cases with a small amount of light component, when an inverted column is found to be optimal, the inverted column may also be an easier solution in practice because the end of the batch can be easily detected from temperature measurements. The temperature in the residue will decrease steadily during the batch (see Fig. 4). For the same separation in the regular column, the end of the batch is difficult to detect based on temperature measurements at the ends of the column alone.

However, there are two main disadvantages with the inverted column. The first is that the reboiler holdup must be very low since the reboiler holdup in an inverted column has the same effect on operating time as the condenser holdup in a regular column, i.e. the operating time will normally increase with reboiler holdup (Mujtaba and Macchietto, 1991). [However, see Sørensen (1994) for cases when this does not hold.] A sufficiently low holdup may be difficult to achieve in practice. Secondly, the holdup in the reboiler must be controlled using the reflux flow from the condenser drum as manipulated variable since the liquid flow from the column section to the reboiler cannot be controlled directly. (In our simulations the liquid flow dynamics were neglected and the level control was assumed perfect.)

\section{COMPARISON OF DYNAMIC BEHAVIOUR}

It is interesting to compare the two columns in terms of dynamic behaviour. We will in the following consider the dynamic responses and the rate of approach to equilibrium under total reflux operation for the regular and the inverted column. We will denote the component taken off as the withdrawn component; i.e. light in the regular column and heavy in the inverted column. The "withdrawal' end of the column is the top for the regular column and the bottom for the inverted column, and the 'feed' end is the bottom for the inverted column and the top for the regular column. Mole fractions are given for the light component unless specifically stated otherwise.
We have considered three different cases under total reflux:

1. The light component is present in only a small amount, $x_{F}=0.1$.

2. The feed is equimolar, $x_{F}=0.5$.

3. The light component is present in a large amount, $x_{F}=0.9$.

Dynamic responses: The total reflux responses for the light component in the feed end, on the trays and in the withdrawal end are shown for the three cases in Fig. 6. Note that the time to reach equilibrium is very short due to the low column and condenser holdups in this example.

The responses for the withdrawal end are the most interesting. We see that in the regular column, the light component is transported upwards from the reboiler and accumulates in the top $\left[x_{D}(\mathrm{reg}) \approx 1\right.$ in the right plots]. In the inverted column the heavy component is transported downwards from the condenser to the bottom $\left[x_{B}(\right.$ inv $) \approx 0$ in the right plots]. Also, by comparing the responses for each column separately, it is found that the dynamic responses are fastest at the withdrawal end for both columns, that is, in the top of the regular column [compare $x_{D}(\mathrm{reg})$ in the right plot with $x_{B}(\mathrm{reg})$ in the left plot] and in the bottom of the inverted column [compare $x_{B}$ (inv) with $x_{D}$ (inv) in the left plot]. This is, however, difficult to see in Fig. 6 due to the scaling used.

Note that the initial responses in Fig. 6 are equal but in opposite direction for the top and bottom of the columns $\left(\mathrm{d} x_{D} /\left.\mathrm{d} t\right|_{t=0}\right.$ in the inverted column equal to $\mathrm{d} x_{B} /\left.\mathrm{d} t\right|_{t=0}$ in the regular column and vice versa).

For the case with equimolar feed, $x_{F}=0.5$ (middle plots), the responses for the regular and inverted column are very similar but not quite equal in terms of being mirror images of each other. This is because the inverted column studied here is not the true inverse of the regular column since the feed is in liquid and not in vapour phase. (This is discussed in Section 5.)

Rate of approach to equilibrium: Next consider the rate of approach to equilibrium. For this comparison the responses are best shown in terms of normalised variables:

$$
x^{\text {norm }}(t)=\frac{x(t)-x^{0}}{x^{s s}-x^{0}}
$$

From Fig. 7 we see that both columns reach equilibrium slowest when the amount of withdrawn component is small, that is when $x_{F}=0.1$ in the regular column $\left[x_{D}(\mathrm{reg})\right.$ in the upper right plot $]$ and $x_{F}=0.9$ in the inverted column $\left[x_{B}\right.$ (inv) in the lower right plot]. The reason for this is that it takes a long time for the small amount of the withdrawn component to move from the feed end through the column, which is filled mostly with the other component, to the withdrawal end. Note that the same effect is also found when the holdup in the column section 
FEED END

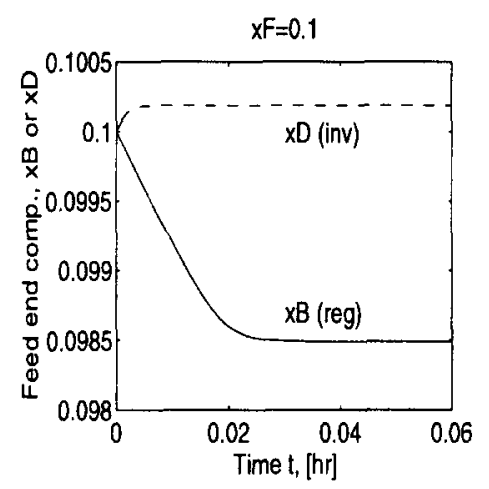

$\mathrm{xF}=0.5$
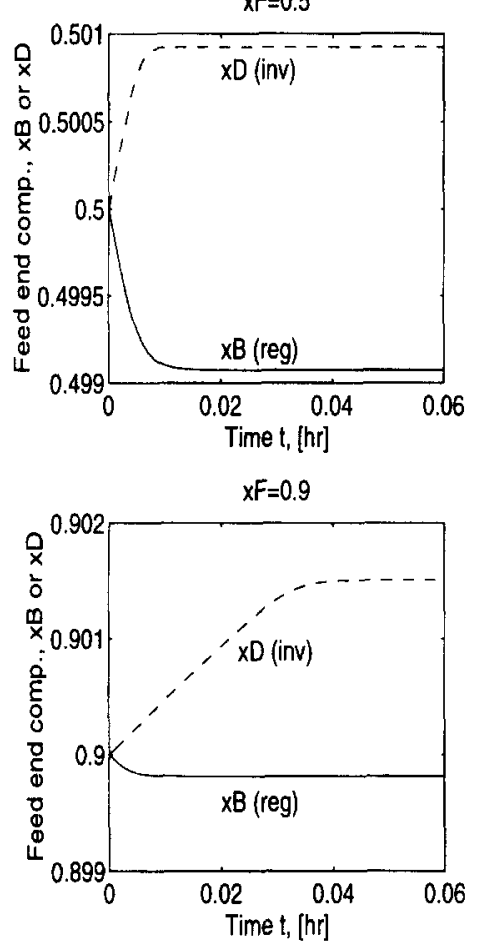

TRAYS

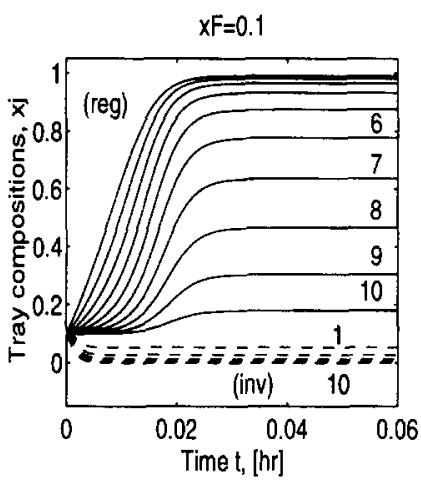

$\mathrm{xF}=0.5$
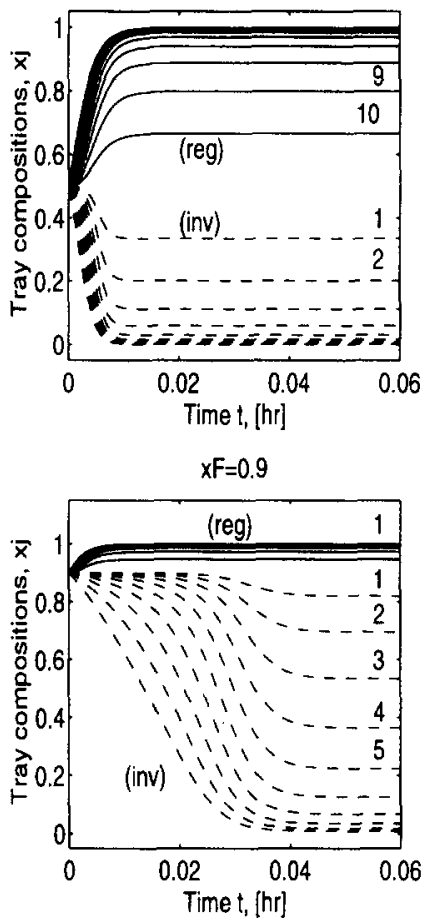

WITHDRAWAL END
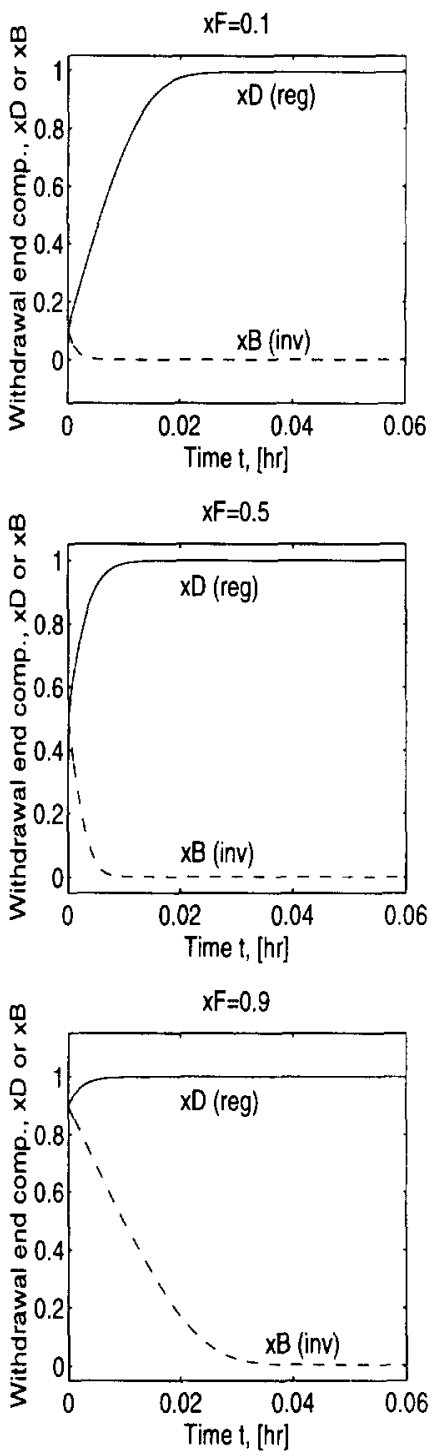

Fig. 6. Time responses of compositions for regular and inverted column under total reflux.

and reboiler/condenser is assumed negligible (not shown).

Note that the fast response in the inverted column coincides with the case where an inverted column is preferable for high-purity separations in terms of minimum operating time $\left(x_{F}=0.1\right)$.

\section{THE PERFORMANCE OF INVERTED SEPARATIONS}

So far we have considered the separation of the same feed mixture in a regular and in an inverted column. It was found that for the case with equimolar feed $\left(x_{F}=0.5\right)$, the dynamic responses for the regular and inverted column are very similar but not quite equal in terms of being mirror images of each other. We will therefore in this section compare what we denote inverted separations: the removal of light com- ponent from a regular column compared to the removal of heavy component from an inverted column or vice versa. The feed compositions (in terms of the light component) are in this case mirror images of each other:

$$
\left.x_{F}(\text { regular column })=1-x_{F} \text { (inverted column }\right) .
$$

This is done in an attempt to more fully understand the behaviour of inverted columns. In practice, the problem one is faced with is the decision as to which column configuration is the best for a given feed mixture, i.e. a given $x_{F}$ in either a regular or an inverted column (or another column configuration).

Let us first go back to the optimal results in Section 3. By comparing the shape of the plots in Fig. 2 it can be seen that the separation in the inverted column 
FEED END
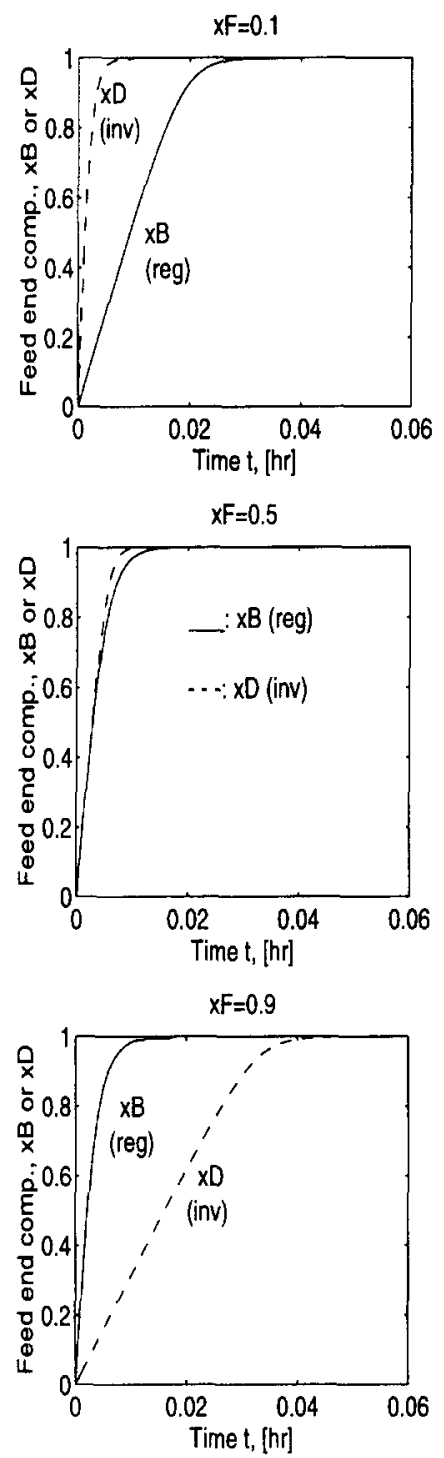

TRAYS
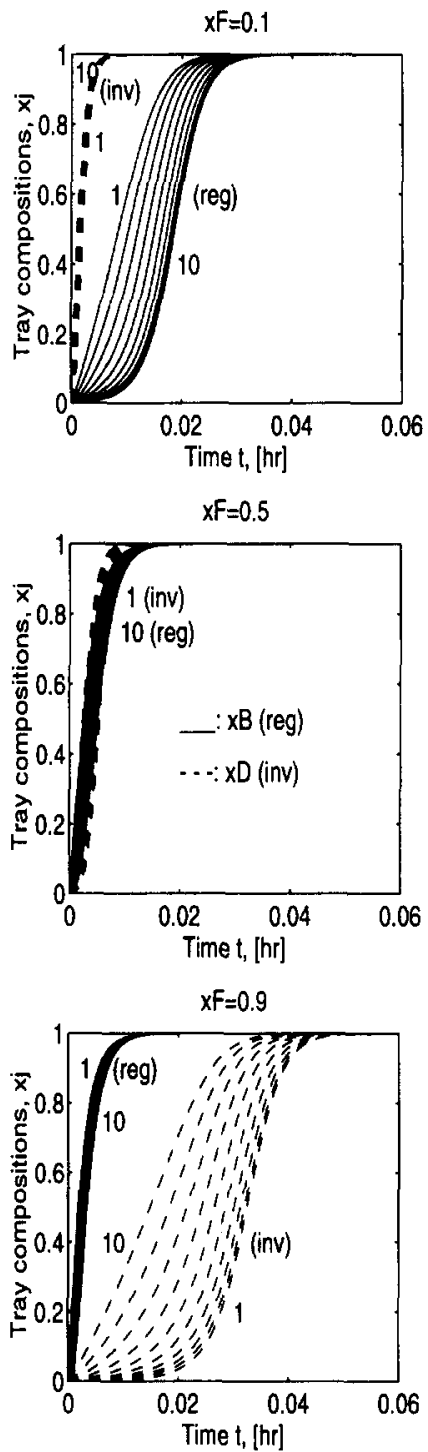

WITHDRAWAL END
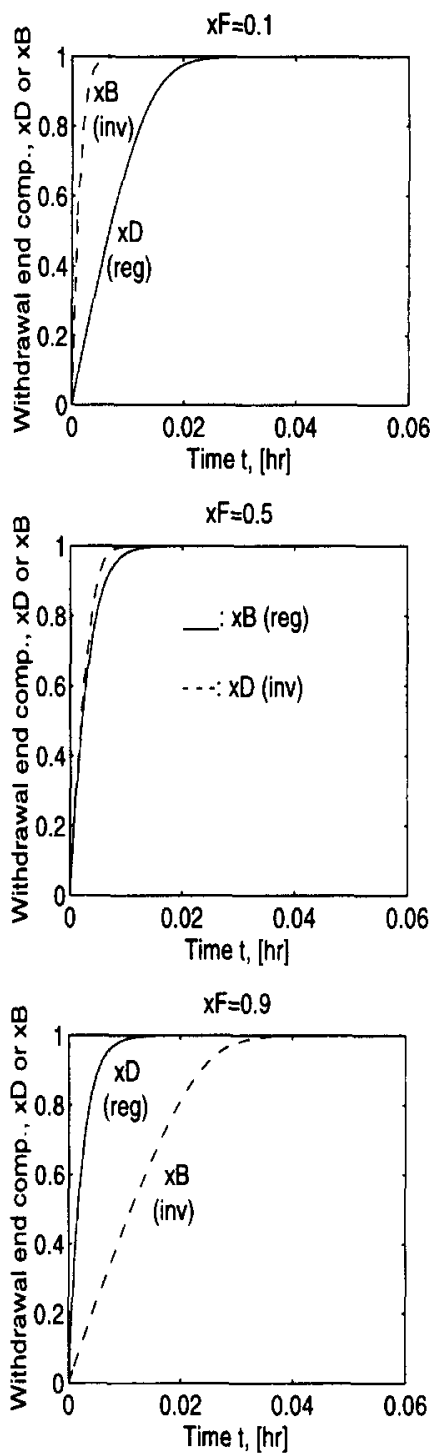

Fig. 7. Time responses for normalised compositions for regular and inverted column under total reflux.

(dotted line in the left plot) has a similar operating time profile as the separation in the regular column (solid line in the right plot). The same comparison can be made for the reverse case. Also, the optimal reflux and reboiler ratios have a similar shape and value for inverted separations (dotted line in the left plot similar to dotted line in the right plot in Fig. 3). Nevertheless, the operating times for inverted separations have different values; for example

inverted column:

$$
t_{f}=2.264 \mathrm{~h} \text { for } x_{F}=0.1 \text { and } x^{\text {spec }}=0.975
$$

regular column:

$$
t_{f}=2.410 \mathrm{~h} \text { for } x_{F}=0.9 \text { and } x^{\text {spec }}=0.975 .
$$

In the following we will compare the regular and the inverted column for inverted separations in terms of dynamic behaviour under total reflux and during production. We will argue that the difference is due to the fact that both the feed and the product are in liquid and not in vapour phase in the inverted column. The inverted column studied so far is therefore not the true inverse of the regular column.

\subsection{Comparison of dynamic behaviour under total re-} flux

In Fig. 8 we have plotted the dynamic responses in composition of the withdrawn component for inverted separation corresponding to the same examples as in Section 4. The withdrawn component is the light component in the regular column and the heavy 
FEED END
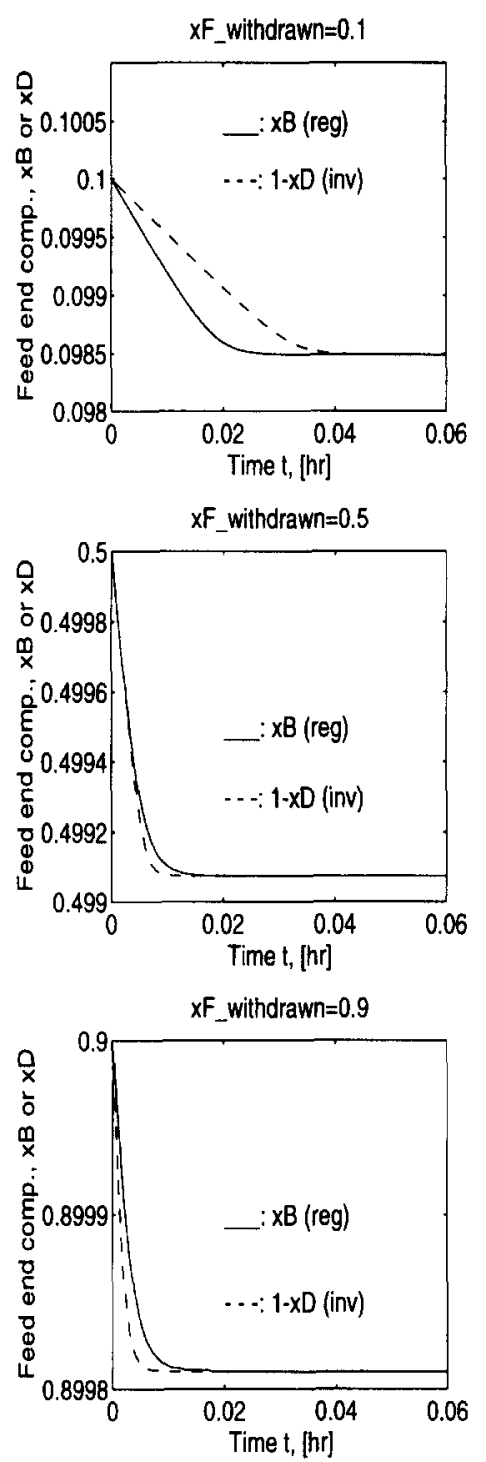

TRAYS

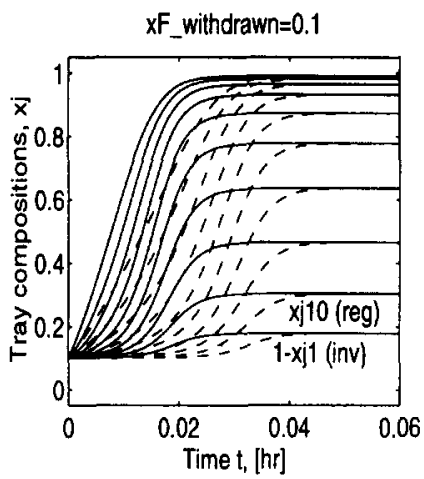

$\mathrm{xF}$ withdrawn $=0.5$

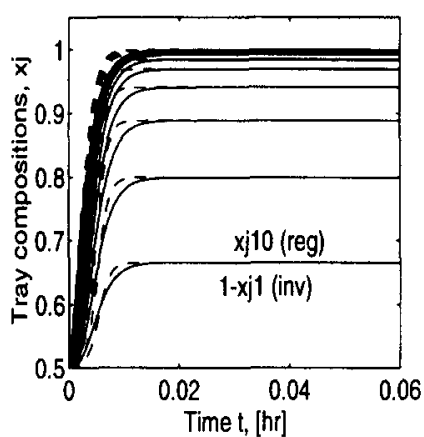

XF withdrawn $=0.9$

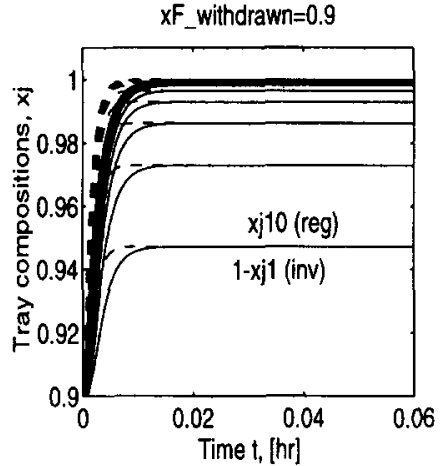

WITHDRAWAL END
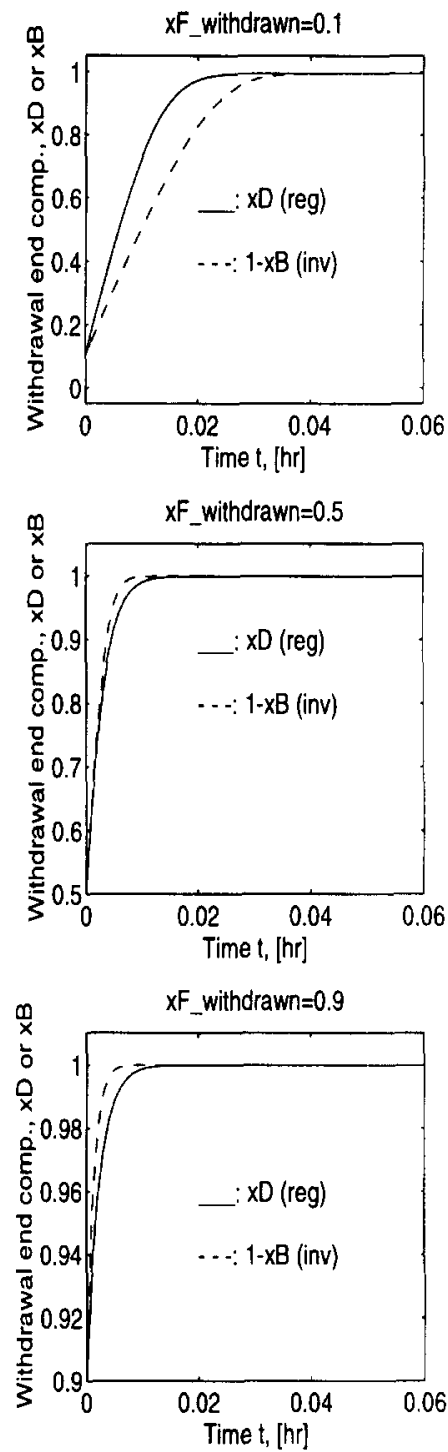

Fig. 8. Time responses of compositions of withdrawn component for a regular and an inverted column under total reflux (inverted separations).

component in the inverted column. The feed composition is given as

$x_{F, \text { withdrawn }}=x_{F, \text { light }}($ regular $)=1-x_{F, \text { light }}($ inverted $)$.

The tray responses (middle plots) are from the bottom and up for increasing light composition for the regular column but from the top and down for increasing heavy composition for the inverted column. We note the following:

1. The steady-state profiles are equal.

2. The approach to equilibrium is different.

The case with a small amount of light component in the regular column is found to approach equilibrium faster than the inverted case with a small amount of heavy component in the inverted column ( $x_{F, \text { withdrawn }}=0.1$, upper plots). However, when the amount of withdrawn component is increased, the rate of approach to equilibrium becomes fastest for the inverted column ( $x_{F \text {, withdrawn }}=0.9$, bottom plots).

\subsection{Operation of inverted separations}

Above we found that the steady-state profiles under total reflux are the same for a regular and an inverted column for a given separation. However, the approach to equilibrium was different. How will this affect the product removal in the two columns? To answer this consider the following two cases:

1. A small amount of withdrawn component is removed from the feed charge $\left(x_{F, \text { light }}=0.1\right.$ for the 
regular column and $x_{F, \text { light }}=0.9$ for the inverted column, i.e. $x_{F \text {, withdrawn }}=0.1$ ).

2. A large amount of withdrawn component is removed from the feed charge $\left(x_{F, \text { light }}=0.9\right.$ for the regular column and $x_{F, \text { light }}=0.1$ for the inverted column, i.e. $x_{F, \text { withdrawn }}=0.9$ ).
The withdrawal and feed end composition profiles are given in Figs 9 and 10 for the two cases. In both cases an initial total reflux period of $0.05 \mathrm{~h}$ is used to reach steady state before the production period starts. Constant product flows are assumed, i.e. $D$ is specified for the regular column and $B$ for the inverted column.
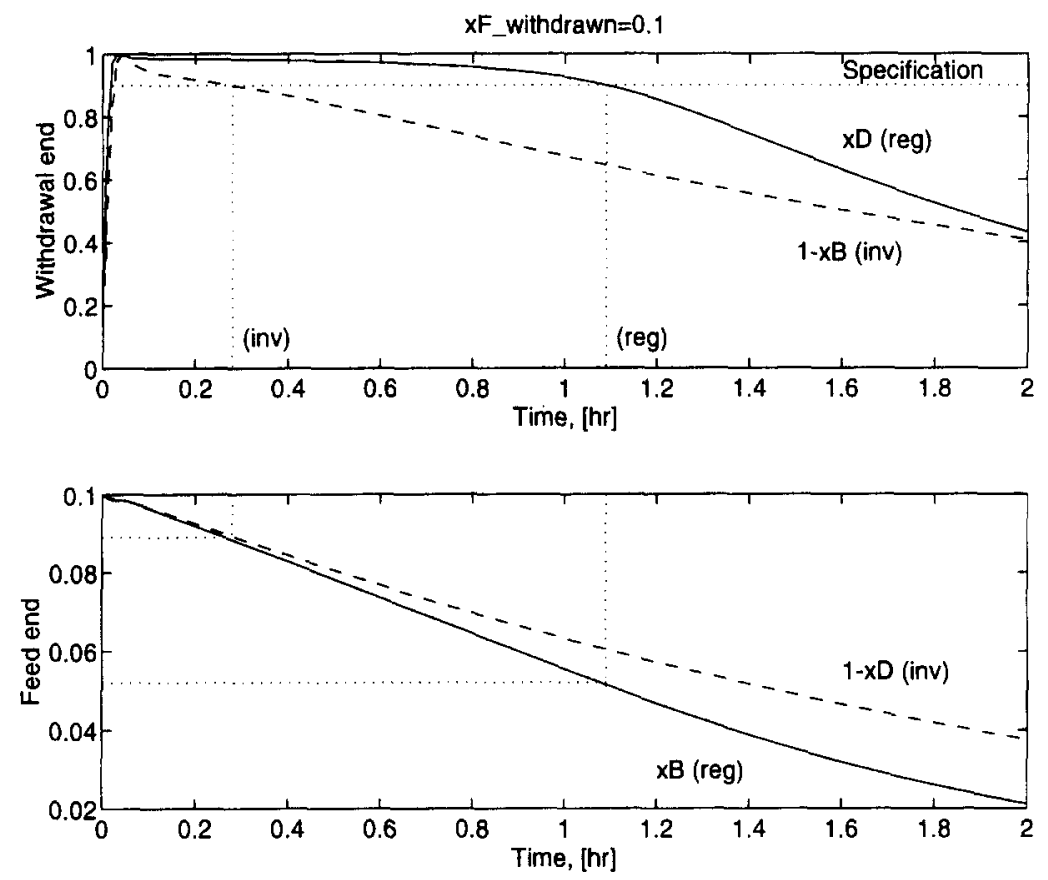

Fig. 9. Withdrawal and feed end composition profiles for the withdrawn component. $\left(x_{F}=0.1\right.$ for the regular column and $x_{F}=0.9$ for the inverted column, $x_{D, \text { light }}^{\text {spec }}($ reg $)=x_{B \text {, heavy }}^{\text {spec }}($ inv $)=0.9$ and $D($ reg $)=$ $B($ inv $)=0.5 \mathrm{kmol} / \mathrm{h}$.)
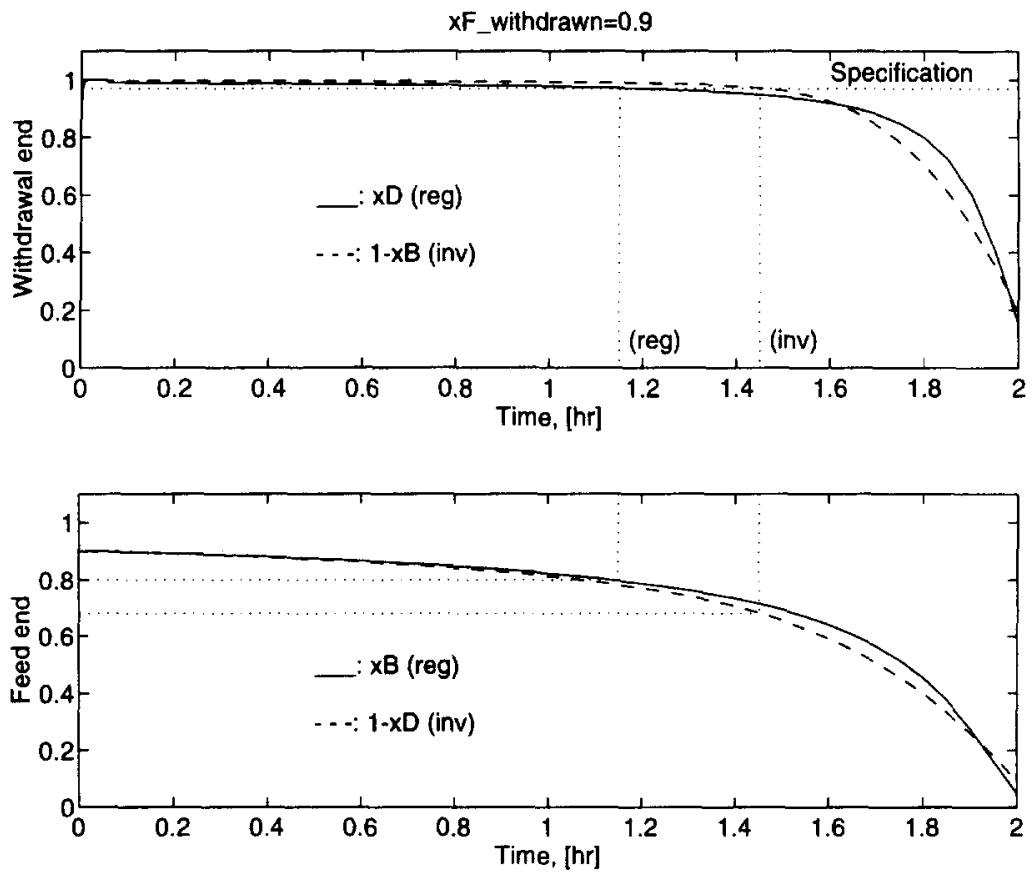

Fig. 10. Withdrawal and feed end compositions for the withdrawn component. $\left(x_{F}=0.9\right.$ for the regular column and $x_{F}=0.1$ for the inverted column, $x_{D, \text { lighi }}^{\text {spec }}(\mathrm{reg})=x_{B, \text { heavy }}^{\text {spec }}($ inv $)=0.97$ and $D(\mathrm{reg})=B($ inv $)=$ $5 \mathrm{kmol} / \mathrm{h}$.) 
The specification for the product composition is for the withdrawn component in both cases, $x_{D, \text { light }}^{\text {spec }}(\mathrm{reg})$ and $x_{B \text {, heavy }}^{\text {spec }}$ (inv). The specification is $x_{D, \text { light }}^{\text {spec }}(\mathrm{reg})=$ $x_{B . \text { heavy }}^{\text {spec }}($ inv $)=x^{\text {spec }}=0.9$ in the first case and $x^{\text {spec }}=0.97$ in the latter.

From Fig. 9 it can be seen that, for the case with a small amount of withdrawn product, the product composition for the inverted column drops below the specification $x_{B \text {, heavy }}^{\text {spec }}=0.9$ very quickly. For the regular column, however, the separation is satisfactory. For this case, the regular column has the fastest responses under total reflux as shown in Fig. 8 top plots.

From Fig. 10 it can be seen that, for the case with a large amount of withdrawn product, the specification for the product composition is held longest for the inverted column. For this case, the inverted column has the fastest responses under total reflux as shown in Fig. 8, bottom plots. However, note that if the product specification had been lower, for example 0.85 , the regular column would have been best.

An explanation for the difference in dynamic behaviour between the two columns can be found by considering the equilibrium and operating lines as suggested by Hasebe et al. (1992). Consider Fig. 11 where the equilibrium and operating lines are plotted for the case with a small amount of withdrawn component (Fig. 9). In Fig. 11, it is assumed that the columns are run with a constant product composition (constant distillate operating policy). Since the compositions are varying (see Fig. 9), a new plot should be made for each instantaneous value of $x_{D}$ or $x_{B}$. However, Fig. 11 still gives a good representation of the separation taking place in Fig. 9.

For the regular column, the product specification $\left(x_{D, \text { light }}^{\mathrm{spec}} \geqslant 0.9\right)$ will be satisfied as long as the feed end composition of the light component is higher than $x_{B, \text { light }}=0.047$. For the inverted column, however, the feed end composition of the heavy component must be higher than $x_{D \text {, heavy }}^{\text {spec }}=0.085$ for the product specification $\left(x_{B \text {, heavy }}^{\text {spec }} \geqslant 0.9\right)$ to be satisfied. This is in agreement with the results in Fig. 9, dotted lines in the bottom plot.

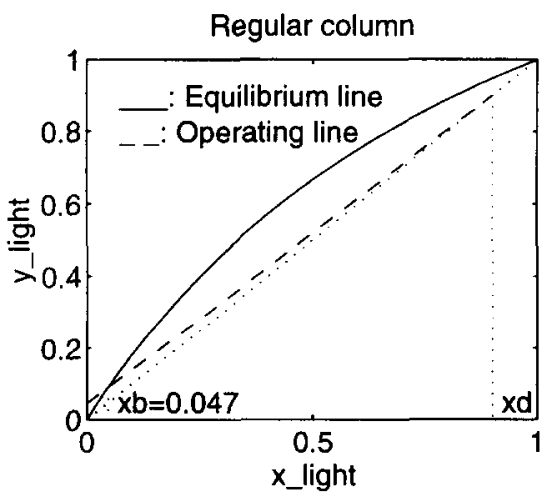

The same discussion on equilibrium and operating lines can be made for the case with a large amount of withdrawn component (not shown).

\subsubsection{Summary. In this section we have found that:}

- The steady-state profiles are equal for inverted separations (light component removal from a regular column compared to heavy component removal from an inverted column).

- The regular column has a faster approach to equilibrium than the inverted column for inverted separations with a small amount of withdrawn component in the feed (light in the regular column and heavy in the inverted column). Also, the regular column is able to maintain a high product composition for a longer period which will again result in either a larger amount of on-spec product or a shorter operating time for this column.

- For inverted separations with a large amount of withdrawn component in the feed (light in the regular column and heavy in the inverted column), the situation is the opposite. A faster approach to equilibrium and a longer on-spec period is found for the inverted column.

Hasebe et al. (1992) explained the difference between regular and inverted columns to be due to the fact that the function $f(x)=y(\alpha, x)-x$, where $y(\alpha, x)$ is the vapour-liquid equilibrium equation, is not symmetrical around the vertical line $x=0.5$. They stated that the regular column always has a better separation efficiency than the inverted column when the relative volatility is constant and the separation conditions are the same (here meaning inverted). However, this is not the case as shown in this section. The main difference between the two columns is due to the fact that the inverted column is not the true inverse of the regular column since the feed and product is in liquid and not in vapour phase.

\subsection{Ideal inverted column}

The true inverse of a regular batch column is denoted an ideal inverted column in the following. It is

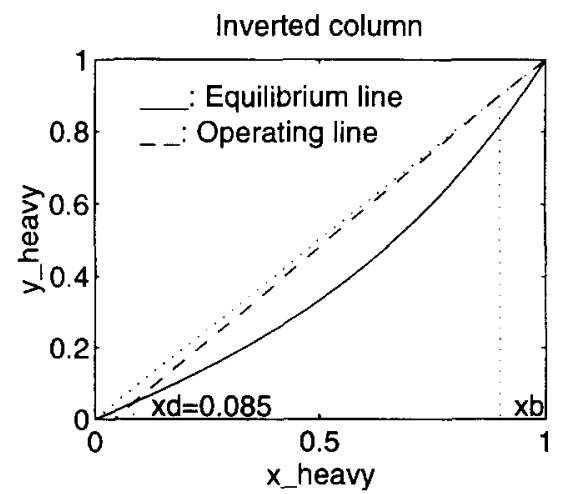

Fig. 11. Equilibrium and operating lines for a regular and an inverted column ( $x_{D \text {. light }}^{\text {spe }}=0.9$ for the regular column and $x_{B \text {, heavy }}^{\text {spec }}=0.9$ for the inverted column). 
a column where the feed is charged as vapour to the condenser drum. This vapour is continuously condensed in a total condenser and fed to the top of the column as liquid. The liquid from the bottom of the column is completely evaporated in the reboiler. A fraction of this vapour is returned to the column and some is taken off as product.

In Fig. 12, a regular column is compared to an ideal inverted column. The column configurations are clearly equivalent provided:

- The liquid feed in the reboiler of the regular column is vapour feed in the condenser of the ideal inverted column.

- The liquid holdup in the condenser of the regular column is vapour holdup in the reboiler of the ideal inverted column.

- The product is taken out as liquid in the top of the regular column but as vapour from the bottom of the ideal inverted column.

- The trays are filled with liquid in the regular column but with vapour in the ideal inverted column.

- The vapour holdup is neglected in the regular column but the liquid holdup is neglected in the ideal inverted column (with our model assumptions).

- The vapour flow is specified in the regular column but the liquid flow is specified in the ideal inverted column (with our model assumptions).

- For constant relative volatility the separation is equivalent provided the liquid mole fraction of light component in the regular column is the mole fraction of heavy component in the inverted column. This follows since

$$
\frac{y_{\text {light }} / x_{\text {light }}}{y_{\text {heavy }} / x_{\text {heavy }}}=\alpha=\frac{x_{\text {heavy }} / y_{\text {heavy }}}{x_{\text {light }} / y_{\text {light }}} .
$$

However, having both the feed charge and the product as vapour will not be very convenient. A more practical solution is therefore to have the feed as liquid in the condenser drum and to remove the product from the reboiler as liquid. This is the column studied so far in this paper and which we denote a practical inverted column. Note that the number of theoretical stages is equal for the regular and the two inverted columns:

\begin{tabular}{lc}
\hline \multicolumn{1}{c}{ Column } & Theoretical stages \\
\hline Regular & $N$ trays + reboiler $=N+1$ \\
Ideal inverted & $N$ trays + condenser $=N+1$ \\
Practical inverted & $N$ trays + reboiler $=N+1$ \\
\hline
\end{tabular}

Note that one may directly obtain operational data for the ideal inverted column from data for a regular batch column. One then needs to reverse the data as follows:

$$
\begin{aligned}
& L^{\mathrm{inv}}=V^{\mathrm{reg}}, \quad y_{F}^{\mathrm{inv}}=1-x_{F}^{\mathrm{reg}}, \quad y_{A}^{\mathrm{inv}}=1-x_{A}^{\mathrm{reg}}, \\
& y_{R}^{\mathrm{inv}}=1-x_{R}^{\mathrm{reg}} .
\end{aligned}
$$

(We use $y_{F}, y_{A}$ and $y_{R}$ to denote that these are vapourphase compositions.)

The data in the right column of Table 1, which are for a regular column with vapour flow $V_{B}=$ $10 \mathrm{kmol} / \mathrm{h}$, will therefore correspond to data for an ideal inverted column with reflux flow $L=10 \mathrm{kmol} / \mathrm{h}$. For example, for the case with $x_{F}=0.9$ and $x_{A}^{\text {spec }}=$ $1-x_{R}^{\text {spec }}=0.99$, the minimum operating time is $3.19 \mathrm{~h}$ when vapour flow is fixed at $V_{B}=10 \mathrm{kmol} / \mathrm{h}$. Thus, with $y_{F}=0.1$ (feed is vapour), $y_{A}^{\text {spec }}=1-y_{R}^{\text {spec }}=0.01$ and reflux flow $L$ fixed at $10 \mathrm{kmol} \mathrm{h}$, the minimum operating time in an ideal inverted column is $3.19 \mathrm{~h}$.

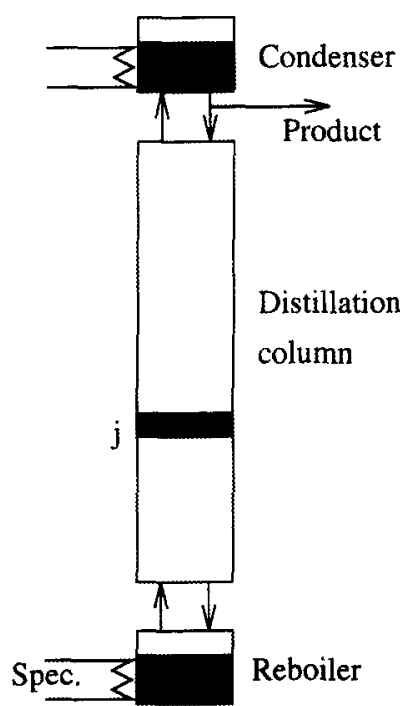

REGULAR COLUMN

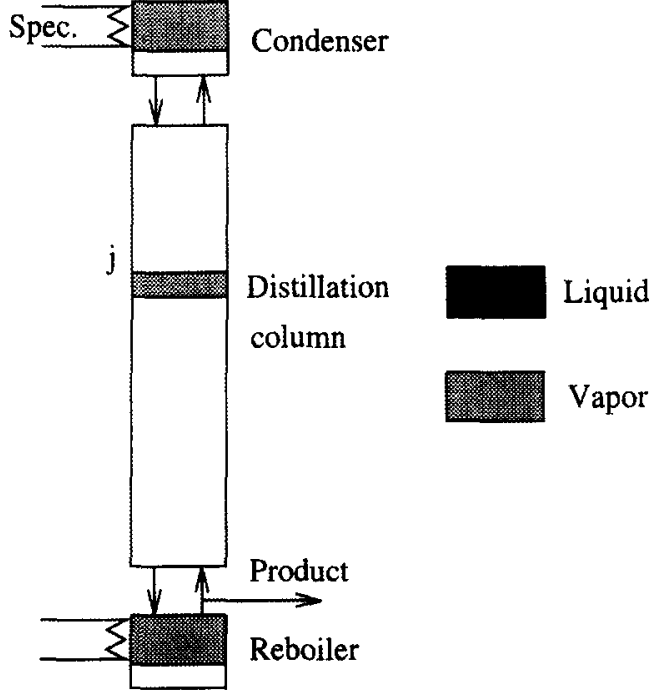

IDEAL INVERTED COLUMN

Fig. 12. A regular and an ideal inverted batch distillation column. 
(If we instead fixed the vapour flow at $10 \mathrm{kmol} / \mathrm{h}$ then the operating time would be even shorter.) It is interesting to note from Table 1 that in a practical inverted column is separation needs $4.04 \mathrm{~h}$, which is significantly higher.

\section{CONCLUSIONS}

In the first part of this paper we compared a regular and inverted column in terms of optimal operation and dynamic behaviour. The inverted column was found to yield the shortest operating time for separations where the light component is present in a small amount in the feed. This is because when light purity is required, it is more time consuming to remove a small amount of light component overhead from a regular column using a very high reflux ratio than to remove a large amount of heavy component from the bottom of an inverted column using a low to moderate reboiler ratio. Also, the dynamic responses are slower in the regular column than in the inverted column for these cases. Note that Robinson and Gilliland (1950), who originally proposed the use of inverted columns, stated that the main advantage was that the light component would be collected in the condenser drum in high purity. This is confirmed in this paper.

In the second part of the paper we found that the steady-state profiles are equal but the approach to equilibrium is different for inverted binary separations (removal of light component in a regular column and of heavy component in an inverted column). This is because the inverted column is not the true inverse of the regular column since the feed and product are in liquid and not in vapour phase.

The use of an ideal inverted column with the feed in vapour phase is, however, more of academical interest. In practice, one is faced with the decision as to whether to use a regular or a practical inverted column configuration for a given feed mixture. We believe that this paper has at least indicated the answer to this question.

\section{NOTATION}

\section{$B$}

D

$H_{A}$

$H_{B}$

$H_{C}$

$H_{F}$

$H_{j}$

$H_{R}$

L

$L_{j}$

$N$

$R$

$R_{B}$ $t$

$t_{0}$

$t_{1}$

$t_{f}$

$V_{j}$

$V_{B}$

$x_{A}$

$x_{B} \quad$ mole fraction of light component in re-

boiler

$x_{D} \quad$ mole fraction of light component in distillate

$x_{F} \quad$ mole fraction of light component in feed

$x_{j} \quad$ mole fraction of light component in

liquid leaving tray $j$

$x_{R} \quad$ mole fraction of light component in residual

$y_{B} \quad$ mole fraction of light component in vapour leaving reboiler

$y_{D} \quad$ mole fraction of light component in vapour distillate

$y_{j} \quad$ mole fraction of light component in vapour leaving tray $j$

\section{Greek letters}

$\alpha \quad$ relative volatility

\section{Superscripts and subscripts}

$0 \quad$ initial conditions

inv inverted column

$j \quad$ tray number

light light component

liq liquid

norm normalised

reg regular column

spec specified value

ss steady state

vap vapour

withdrawn withdrawn component (light in regular and heavy in inverted column)

\section{REFERENCES}

Chiotti, O. J. and Iribarren, O. A., 1991, Simplified models for binary batch distillation. Comput. Chem. Engng 15, $1-5$.

Chiotti, O. J., Salomone, H. E. and Iribarren, O. A., 1993, Selection of multicomponent batch distillation sequences. Chem. Engng Commun. 119, 1-21.

Hasebe, S., Abdul Aziz, B., Hashimoto, I. and Watanabe, T., 1992, Optimal design and operation of complex batch distillation column. Proceedings of the IPAC Workshop, London.

Mujtaba, I. M. and Macchietto, S., 1991, The role of holdup on the performance of binary batch distillation. Proceedings PSE '91, Montebello, Canada, pp. 1.19.1-1.19.15.

Mujtaba, I. M. and Macchietto, S., 1992, Optimal operation of reactive batch distillation. AIChE 1992 Annual Meeting, Miami Beach.

Mujtaba, I. M. and Macchietto, S., 1994, Optimal operation of multicomponent batch distillation. A comparative study using conventional and unconventional columns. Proceedings ADCHEM '94, Japan. 
Robinson, C. S. and Gilliland, E. R., 1950, Elements of Fractional Distillation, 4th Edn. McGraw-Hill, New York. Sørensen, E., 1994, Studies on optimal operation and control of batch distillation columns. Ph.D. Thesis, University of Trondheim-NTH, Trondheim.

SPEEDUP Release 5.4 User Manual, 1993, Prosys Technology Ltd.

Vassiliadis, V., 1993, Computational solution of dynamic optimization problems with general differential-algebraic constraints. Ph.D. Thesis, University of London, London.

\section{APPENDIX: DYNAMIC MODELS}

The dynamic models used in this study are valid under the following assumptions:

(1) Staged batch distillation column (trays numbered from the top and down).
(2) Perfect mixing on all trays

(3) Negligible vapour holdup.

(4) Constant stage pressures and tray efficiencies.

(5) Equal vapour flows in the column.

(6) Total condensation with no subcooling in the condenser.

(7) Constant relative volatility.

(8) Constant molar liquid holdup on all trays (liquid dynamics are neglected).

(9) Constant molar condenser holdup in the regular column (perfect control).

(10) Constant molar reboiler holdup in the inverted column (perfect control)

The following differential and algebraic equations result (see Fig. 1):

\begin{tabular}{|c|c|c|}
\hline & Regular column & Inverted column \\
\hline Reboiler & $\begin{aligned} \mathrm{d} \mathbf{H}_{B} / \mathrm{d} \mathbf{t} & =L_{N}-V_{B} \\
\mathrm{~d}\left(\mathbf{H}_{B} \mathbf{x}_{B}\right) / \mathrm{d} \mathbf{t} & =L_{N} x_{N}-V_{B} y_{B}\end{aligned}$ & $\begin{array}{c}\mathbf{0}=L_{N}-V_{B}-\mathbf{B} \\
\mathbf{H}_{B} \mathrm{~d} \mathbf{x}_{B} / \mathrm{d} \mathbf{t}=L_{N} x_{N}-V_{B} y_{B}-\mathbf{B} \mathbf{x}_{B} \\
\mathbf{R}_{B}=\mathbf{V}_{B} / \mathbf{L}_{N}\end{array}$ \\
\hline Column tray & $\begin{array}{l}0=L_{j-1}+V_{j+1}-L_{j}-V_{j} \\
H_{j} \mathrm{~d} x_{j} / \mathrm{d} t=L_{j-1} x_{j-1} \\
\quad+V_{j+1} y_{j+1}-L_{j} x_{j}-V_{j} y_{j} \\
\quad V_{j}=V_{j+1}\end{array}$ & $\begin{array}{l}0=L_{j-1}+V_{j+1}-L_{j}-V_{j} \\
H_{j} \mathrm{~d} x_{j} / \mathrm{d} t=L_{j-1} x_{j-1} \\
\quad+V_{j+1} y_{j+1}-L_{j} x_{j}-V_{j} y_{j} \\
\quad V_{j}=V_{j+1}\end{array}$ \\
\hline Condenser & $\begin{array}{c}\mathbf{0}=V_{1}-L-\mathbf{D} \\
\mathbf{H}_{C} \mathrm{~d} x_{D} / \mathrm{d} \mathbf{t}=V_{1} y_{1}-L x_{D}-\mathbf{D} \mathbf{x}_{\mathbf{D}} \\
\mathbf{R}=\mathbf{L} / \mathbf{V}_{1}\end{array}$ & $\begin{aligned} \mathrm{d} \mathbf{H}_{C} / \mathrm{d} \mathbf{t} & =V_{1}-L \\
\mathrm{~d}\left(\mathbf{H}_{C} \mathbf{x}_{D}\right) / \mathrm{d} \mathbf{t} & =V_{1} y_{1}-L x_{D}\end{aligned}$ \\
\hline Accumulator & $\begin{array}{c}\mathrm{d} H_{A} / \mathrm{d} t=\mathbf{D} \\
\mathrm{d}\left(H_{A} x_{A}\right) / \mathrm{d} t=\mathbf{D} \mathbf{x}_{D}\end{array}$ & $\begin{array}{c}\mathrm{d} H_{A} / \mathrm{d} t=\mathbf{B} \\
\mathrm{d}\left(H_{A} x_{A}\right) / \mathrm{d} t=\mathbf{B x}_{B}\end{array}$ \\
\hline Equilibrium & $y_{j}=\frac{\alpha x_{j}}{1+(\alpha-1) x_{j}}$ & $y_{j}=\frac{\alpha x_{j}}{1+(\alpha-1) x_{j}}$ \\
\hline
\end{tabular}

The reflux ratio $R$ for the regular column is the internal reflux ratio $L / V_{1}$, not the usual external ratio $L / D$. (This is better solved numerically since $R_{\text {internal }} \in[0,1]$ whereas $R_{\text {external }} \in[0, \infty]$.) The reboiler ratio in the inverted column, $R_{B}$, is also defined as an internal ratio $V_{B} / L_{N}$. The external ratio is in this case given as $R_{B \text {, external }}=V_{B} / B$.

It is assumed that the vapour flow $V_{B}$ can be manipulated directly. The heavy product flow $B$ in the inverted column is assumed to be taken out from the reboiler as liquid with a composition equal to $x_{B}$. 